\title{
REVIEW
}

\section{Balancing Antiplatelet and Anticoagulant Therapies in Patients with Cardiovascular Disease}

\author{
Edward T. Carreras · Jessica L. Mega
}

To view enhanced content go to www.cardiologytherapy-open.com

Received: February 4, 2013 / Published online: April 25, 2013

(C) The Author(s) 2013. This article is published with open access at Springerlink.com

\section{ABSTRACT}

Anticoagulation is needed for stroke prevention in patients with atrial fibrillation. Antiplatelet therapy is essential for the prevention of stent thrombosis and the reduction of cardiovascular events in patients who undergo coronary stenting and suffer acute coronary syndromes. When these conditions overlap, the individual antithrombotic strategies are commonly combined, and the efficacy benefit of triple oral antithrombotic therapy is assumed to outweigh the bleeding risk based on the available data. Recent studies have investigated this topic further, including the first randomized controlled trial to address this issue. This new evidence challenges previous

E. T. Carreras $(\bowtie) \cdot$ J. L. Mega

Brigham and Women's Hospital, Harvard Medical School, 75 Francis St, Boston, MA 02115, USA

e-mail: ecarreras@partners.org

J. L. Mega

e-mail: jmega@partners.org

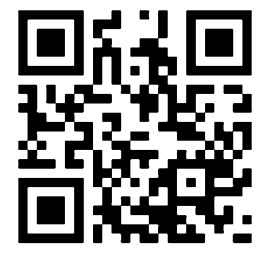

Enhanced content for this article is

available on the journal web site:

www.cardiologytherapy-open.com assumptions and may have implications for future practice and investigation.

Keywords: Antiplatelet therapy and anticoagulation; Coronary stenting and atrial fibrillation; Triple oral antithrombotic therapy; Triple therapy; WOEST trial

\section{INTRODUCTION}

Of the roughly 15.4 million Americans with coronary artery disease (CAD), approximately $4.6 \%$ of them suffer an acute coronary syndrome (ACS) each year and 3.2\% undergo percutaneous coronary intervention (PCI) [1]. Of those who undergo PCI, 5-7\% have a preexisting indication for chronic oral anticoagulation (OAC) [2]. These patients require antithrombotic strategies that optimally balance the competing risks of bleeding, stroke, and stent thrombosis.

Prior to the common use of dual antiplatelet therapy (DAPT), approximately $6-24 \%$ of patients suffered stent thrombosis in the first year after stent placement, with a mortality rate up to $50 \%$ [3]. With the use of routine DAPT after PCI, the incidence of stent thrombosis has 
averaged roughly $1-2 \%$ over the first year after PCI with a mortality rate of $10-20 \%$ [2]. OAC is equally important in atrial fibrillation (AF). The annual risk of stroke ranges $2-18 \%$ depending on patient risk factors, and warfarin provides a relative risk reduction of approximately $60 \%$, versus just $20 \%$ for aspirin, as compared to control [4-6].

There is a paucity of evidence regarding the optimal antithrombotic strategy for patients on chronic OAC who undergo PCI. While triple oral antithrombotic therapy (TOAT) is often prescribed to maximize reductions of stroke and stent thrombosis, there is concern that the increased bleeding risk may outweigh the assumed efficacy benefit. Until recently, only a few formal recommendations for antithrombotic therapies in these patients have been published, and these recommendations have been based mostly on retrospective data and older randomized trials that studied the addition of warfarin to aspirin in ACS. Based on these data, current common practice is to use TOAT for at least a short period of time after PCI depending on patient risk factors. Recently, new evidence has emerged suggesting that other strategies may be preferred, including a randomized controlled trial (RCT) to address this topic. This review will briefly summarize the available evidence and guidelines and focus on reviewing the newer studies.

\section{MATERIALS AND METHODS}

This review article incorporates data from observational studies, review articles, available guidelines, as well as a randomized controlled trial on the use of TOAT in patients with AF and CAD. Relevant studies were obtained using a MEDLINE search for triple antithrombotic therapy, AF, and PCI. In addition, randomized controlled trials of the use of anticoagulation and single antiplatelet therapies (SAPT) in patients with CAD were also included. Studies were chosen based on their inclusion and influence on current guidelines and practice.

\section{DISCUSSION}

\section{Stroke Prevention in AF}

AF is a common condition, with a prevalence of approximately $1 \%$ and a lifetime risk of approximately $25 \%$ after the age of $40[5,7]$. Stroke prevention in AF is one of the most common indications for chronic OAC, which has been found to be superior to both single and dual antiplatelet therapies at reducing stroke [3]. Using the available evidence of the relative efficacy and safety of warfarin, SAPT with aspirin, and DAPT with aspirin and clopidogrel, clinicians are able to tailor stroke prevention strategies to their individual patients based on their risk factors. Table 1 depicts the relative efficacy and safety of these common antithrombotic strategies in $\mathrm{AF}$, derived from the American College of Chest Physicians (ACCP) guidelines for antithrombotic therapy for $\mathrm{AF}$ and based on available data from several studies and on a number of broad assumptions [3]. For most patients with at least one risk factor for stroke, the efficacy benefit of anticoagulation sufficiently outweighs the bleeding risk and is the most appropriate choice.

\section{Antiplatelet Therapy After PCI}

Antithrombotic therapy is necessary to prevent recurrent ischemic events and stent thrombosis after ACS and/or PCI. Aspirin has been long known to significantly reduce cardiovascular events after ACS [8]. Over the past decade, DAPT 
Table 1 Comparison of the efficacy and safety of antithrombotic agents in atrial fibrillation

\begin{tabular}{lllc}
\hline & $\begin{array}{l}\text { Death, relative } \\
\text { effect }(\mathbf{9 5 \%} \mathbf{C I})\end{array}$ & $\begin{array}{l}\text { Non-fatal stroke, } \\
\text { relative effect } \\
\mathbf{( 9 5 \% ~ C I )}\end{array}$ & $\begin{array}{l}\text { Non-fatal major } \\
\text { extracranial bleeds, } \\
\text { relative effect (95\% CI) }\end{array}$ \\
\hline Warfarin versus no Tx & $0.72(0.55-0.94)$ & $0.34(0.23-0.49)$ & $2.58(1.12-5.97)$ \\
ASA versus no Tx & $0.89(0.75-1.05)$ & $0.79(0.65-0.96)$ & $1.60(1.40-1.80)$ \\
Warfarin versus ASA & $0.97(0.85-1.12)$ & $0.48(0.33-0.70)$ & $1.42(0.89-2.29)$ \\
Warfarin versus ASA + clopidogrel & $0.98(0.79-1.22)$ & $0.56(0.39-0.82)$ & $0.91(0.67-1.23)$ \\
ASA + clopidogrel versus ASA alone & $0.98(0.90-1.07)$ & $0.72(0.61-0.85)$ & $1.50(1.18-1.89)$ \\
\hline
\end{tabular}

$A S A$ aspirin, $C I$ confidence interval, $T x$ treatment

has been studied extensively in ACS and elective PCI and found to significantly improve outcomes when compared to aspirin alone, with the majority of studies focusing on clopidogrel as the second antiplatelet agent [9, 10]. Prasugrel and ticagrelor have been the subject of more recent investigation; these agents generally achieve higher degrees of platelet inhibition than clopidogrel, do not appear to be affected by CYP2C19 polymorphisms, and have been associated with reductions in cardiovascular events after ACS with an associated increase in nonprocedure related bleeding $[11,12]$.

Current American College of Cardiology Foundation/American Heart Association/ Society for Cardiovascular Angiography and Interventions (ACCF/AHA/SCAI) guidelines recommend at least 12 months of DAPT after PCI. Several published and ongoing trials have compared short-term (6-12 months) versus long-term (>12 months) DAPT after PCI; while the design and results of these individual trials can be debated and cross-trial comparisons are challenging, the overall results suggest a trend towards a lack of benefit of longer-term DAPT, regardless of the type of stent used [13-16]. Several ongoing trials are exploring this concept further; any change in the recommendations for DAPT duration in the future will have a notable impact on recommendations for triple therapy in patients on OAC undergoing PCI.

\section{Anticoagulation After ACS}

The use of warfarin after ACS has been studied in several RCTs and meta-analyses [17, 18]. In the Warfarin-Aspirin Reinfarction Study (WARIS II), 3,630 patients with acute myocardial infarction (MI) were randomized to either aspirin $160 \mathrm{mg}$, warfarin with an international normalized ratio (INR) target of 2.8-4.2, or aspirin $75 \mathrm{mg}$ in combination with warfarin with an INR target of 2.0-2.5 [19]. When compared to aspirin $160 \mathrm{mg}$, warfarin (rate ratio, 0.81; 95\% confidence interval [CI], $0.69-0.95 ; P=0.03)$ and warfarin plus aspirin (rate ratio, $0.71 ; 95 \%$ CI $0.60-0.83 ; P=0.001$ ) produced a significant reduction in the composite primary end point of death, nonfatal recurrent MI, or thromboembolic stroke. Both treatments also lead to significantly more major, non-fatal bleeding when compared to aspirin $160 \mathrm{mg} \quad(P<0.001)$. The two anticoagulation arms did not differ significantly with respect to either endpoint.

The Anti-thrombotics in the Secondary Prevention of Events in Coronary Thrombosis-2 (ASPECT-2) trial randomized patients to three similar arms and showed similar results to WARIS 
II [20]. When compared to aspirin, high-intensity anticoagulation (hazard ratio [HR], 0.55; 95\% CI 0.30-1.00; $\quad P=0.0479)$ and aspirin plus moderate-intensity anticoagulation (HR 0.50; 95\% CI 0.27-0.92; $P=0.03$ ) lead to a significant reduction in the primary endpoint of death, MI, or stroke. There was no significant difference between the two anticoagulation groups with respect to the primary endpoint.

Based on the studies of warfarin in ACS, driven mostly by the results of WARIS II and ASPECT-2, warfarin was at least as efficacious as aspirin at reducing the risk of death, recurrent MI, and stroke after ACS (Figs. 1, 2) [17]. Furthermore, these studies demonstrate that warfarin alone appears to be sufficient as single therapy, and the addition of aspirin to warfarin was not found to have an additional benefit at reducing the composite endpoint of recurrent MI, stroke, or death. However, patients in these studies were not treated with modern interventional therapies, such as drugeluting stents. As such, caution should be taken in applying these results to current practice.

\section{Evidence and Guidelines for TOAT}

A few consensus documents have been published with recommendations for TOAT, the bases for which are mostly derived from small observational studies [3, 21-26]. The available observational data show that patients receiving TOAT experienced a roughly threefold higher risk of major bleeding than did patients receiving DAPT, while the shorter versus longer durations of TOAT were associated with a twofold lower risk of major bleeding [27]. In patients with previous indications for OAC who underwent PCI, DAPT with suspension of OAC was associated with a threefold increased risk of thromboembolic events, while TOAT was associated with a lower risk of stent thrombosis and MI [27]. When analyzed in aggregate, these studies show that patients with AF who undergo PCI and suspend OAC during DAPT suffer an increased incidence of thromboembolic events when compared to either TOAT or OAC plus SAPT, while the risk of major bleeding is increased [27].

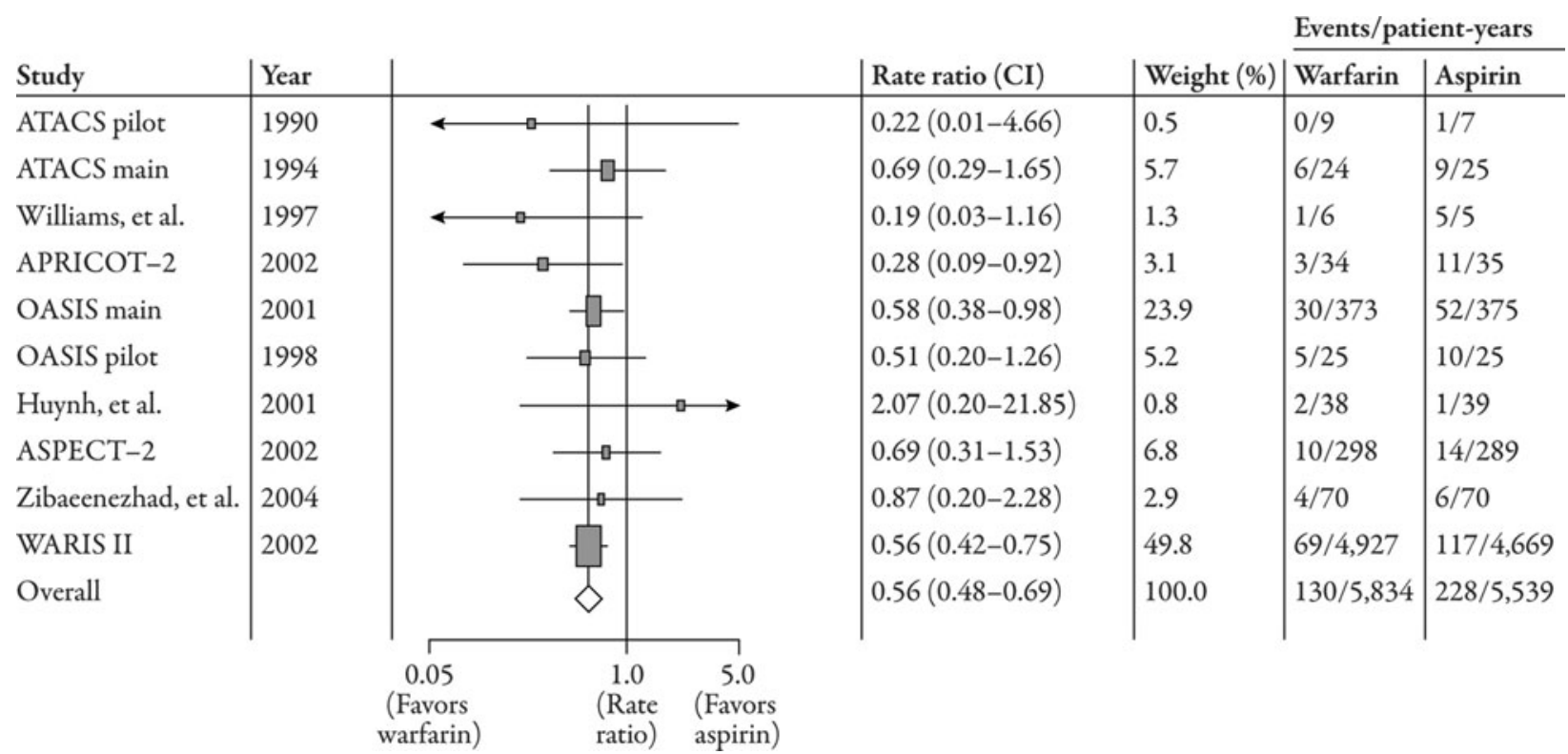

Fig. 1 Rate ratios of recurrent myocardial infarction for warfarin plus aspirin compared with aspirin alone. Figure adapted using data from Rothberg et al. [17] 


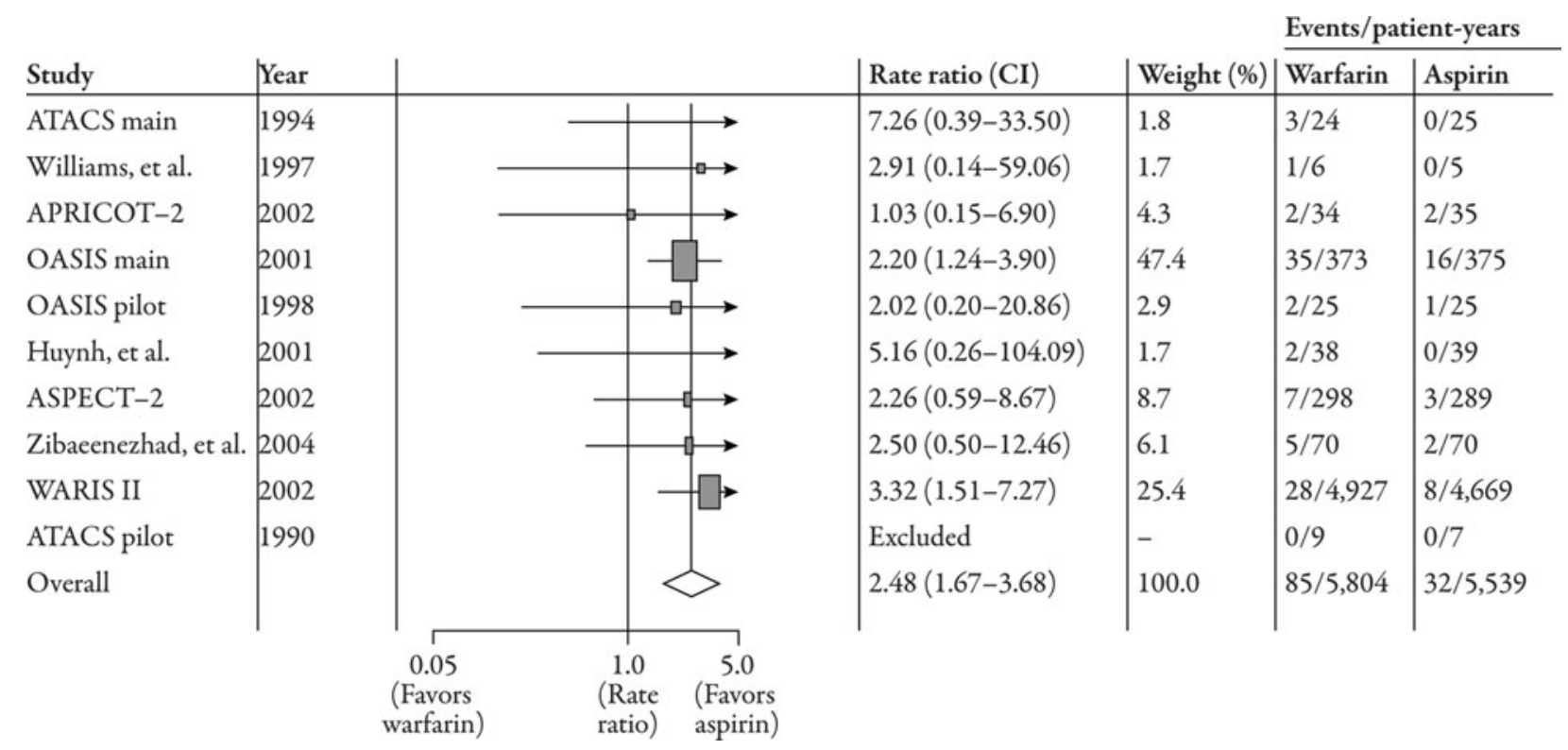

Fig. 2 Rate ratios of major bleeding for warfarin plus aspirin compared with aspirin alone. Figure adapted using data from Rothberg et al. [17]

The European Society of Cardiology (ESC) Working Group on Thrombosis makes some recommendations for the management of patients with AF who have ACS and/or undergo PCI (Table 2) [28]. In general, they advise not to stop anticoagulation and to use TOAT for a short period of time after PCI, the duration of which depends on bleeding risk and stent type. In addition, they recommended using bare metal stents, targeting an INR range 2.0-2.5 for patients receiving TOAT, and using radial access during PCI.

The ACCP published clinical practice guidelines for the management of antithrombotic therapy in patients with atrial fibrillation, including those with $\mathrm{AF}$ and $\mathrm{CAD}$ (Table 3) [3]. The authors felt that the available retrospective data were too prone to bias and instead based their recommendations on the studies of warfarin in ACS. They made several assumptions in extrapolating these data, specifically that the addition of clopidogrel to both study arms, aspirin plus warfarin and aspirin alone, would not change the relative impacts of those treatments on death, non-fatal MI, and non-fatal extracranial bleeding. Their recommendations ultimately differ slightly from those of the ESC in that they indicate that OAC can be safely stopped for some patients at low risk of stroke while on DAPT after PCI.

Another set of recommendations was recently published in Circulation: Cardiovascular Interventions (Fig. 3) [2]. For patients at very low risk of stroke, the authors advise omitting OAC after PCI. For all other patients, they recommend TOAT for 1-6 months depending on risks of bleeding and stent thrombosis and stent type.

\section{Recent Studies}

Published in September, 2012, a retrospective analysis of 11,480 patients in Denmark registries studied the effect of multiple antithrombotic strategies used in $\mathrm{AF}$ after 


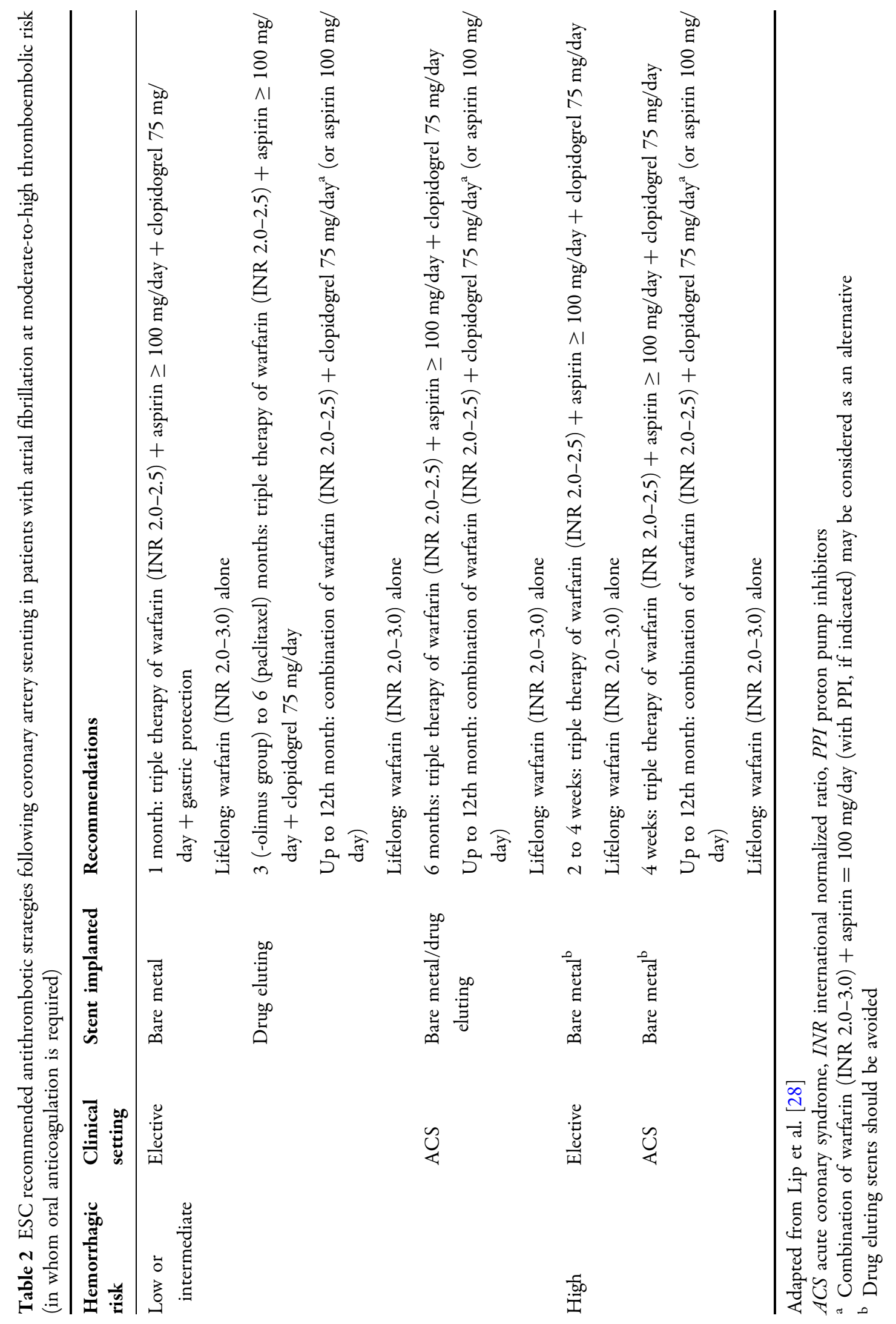


Table 3 ACCP guidelines

\begin{tabular}{llll}
\hline Stroke risk & Clinical setting & Type of stent & Recommendation \\
\hline $\mathrm{CHADS}_{2} 0-1$ & Elective or ACS & BMS or DES & $0-12$ mos: DAPT \\
& & & After 12 mos: OAC \\
$\mathrm{CHADS}_{2}>1$ & Elective & BMS & $0-1$ mo: TOAT \\
& & $1-12$ mos: OAC + SAPT \\
& DES & After 12 mos: OAC \\
& & $0-6$ mos: TOAT \\
& & $6-12$ mos: OAC + SAPT \\
\end{tabular}

ACCP guidelines for the management of antithrombotic therapy in patients with atrial fibrillation after coronary stenting $A C C P$ American College of Chest Physicians, BMS bare metal stent, DES drug-eluting stent, mos months, $O A C$ oral anticoagulant, $S A P T$ single antiplatelet therapy, TOAT triple oral antithrombotic therapy

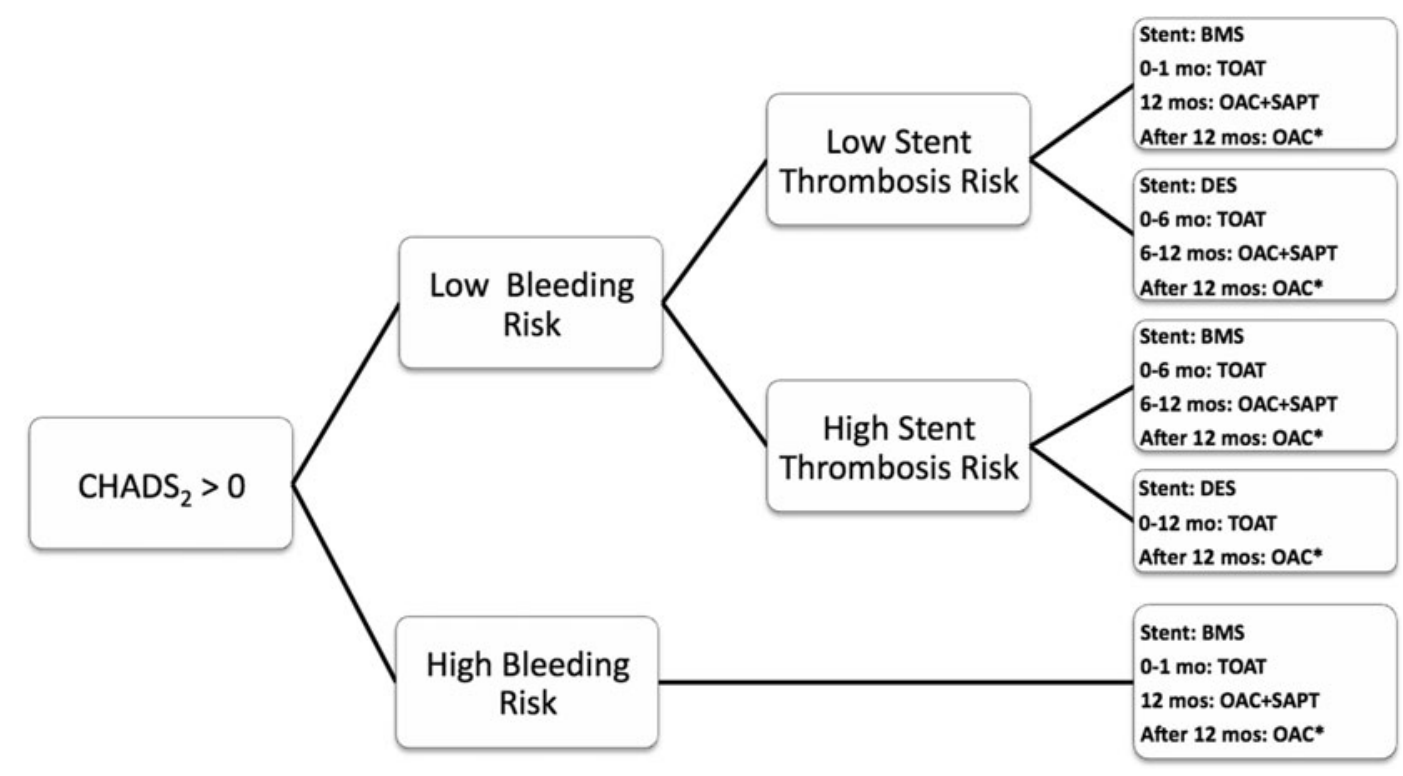

Fig. 3 Circulation guidelines for the management of antithrombotic therapy in patients with atrial fibrillation after coronary stent placement. Asterisk In patients at high risk for atherothrombotic events including stent thrombosis, continued single antiplatelet therapy with warfarin

coronary intervention [29]. The authors found that TOAT was associated with more bleeding than vitamin $\mathrm{k}$ antagonist (VKA) plus SAPT at 90 days (HR 1.47; 95\% CI 1.04-2.08) and 1 year (HR 1.36; 95\% CI 0.95-1.95). With regard to should be considered after 12 months. BMS bare metal stent, DES drug-eluting stent, mos months, $O A C$ oral anticoagulation, $S A P T$ single antiplatelet therapy, TOAT triple oral antithrombotic therapy

efficacy, TOAT and VKA plus SAPT were statistically similar (HR 1.15; 95\% CI 0.95-1.40) and superior to all other strategies, suggesting that VKA plus SAPT might be preferred to TOAT. 
The What is the Optimal antiplatElet and anticoagulant therapy in patients with oral anticoagulation and coronary StenTing (WOEST) trial is a completed RCT to study the comparison of TOAT versus VKA plus SAPT [30]. The trial design and rationale were published in 2009, and the study was published in February, 2013 [30-32]. The authors randomized 573 patients undergoing PCI in an open-label, intention-to-treat design to either double therapy (warfarin and clopidogrel $75 \mathrm{mg}$ ) or triple therapy (warfarin, clopidogrel $75 \mathrm{mg}$, and aspirin $80 \mathrm{mg}$ ) in order to test the hypothesis that double therapy is superior to triple therapy with respect to bleeding. Study patients were free of any thrombolysis in myocardial infarction (TIMI) major bleeding in the past 1 year and had an indication for OAC for at least 1 year after PCI. Approximately, $70 \%$ of patients required OAC for AF; mechanical valves accounted for approximately $10 \%$. The primary endpoint was the composite of all TIMI bleeding; the secondary endpoints included the composite of death, MI, stroke, systemic embolism, target vessel revascularization and stent thrombosis, as well as the individual components of the composite endpoints.

At 1 year, the cumulative incidence of all TIMI bleeding, which included major, minor, and minimal events, was $44.4 \%$ in the triple therapy group compared to $19.4 \%$ with double therapy (HR 0.36; 95\% CI 0.26-0.50; $P<0.0001$ ). The results were driven by differences in TIMI minimal and minor bleeding, with a nonsignificant trend towards a reduction in TIMI major bleeding seen with double therapy. There was no difference in intracranial bleeding between the two groups. The primary endpoint differences were consistent across old age, gender, ACS, indication for OAC, and stent type.

The composite of death, MI, stroke, systemic embolism, target vessel revascularization, and stent thrombosis occurred in $17.6 \%$ of patients receiving triple therapy compared to $11.1 \%$ receiving double therapy (HR 0.56; 95\% CI $0.35-0.91 ; \quad P=0.025)$. This difference was driven by a reduction in all-cause mortality, with one-year rates of $6.3 \%$ versus $2.5 \%$ in triple and double therapy, respectively (HR 0.39; 95\% CI $0.16-0.93 ; P=0.027)$. Rates of MI, stroke, and stent thrombosis were numerically lower in the double therapy group without statistical significance.

\section{Integrating New Data into Practice}

As previously discussed, several of the trials that studied the use of warfarin in ACS, such as WARIS II and ASPECT-2, included arms that compared warfarin alone versus warfarin plus aspirin and found no statistically significant difference in efficacy [17]. These results set a precedent to suggest that the addition of aspirin to therapeutic anticoagulation does not add significantly to the prevention of death, nonfatal MI, or stroke after ACS; however, notably, there were low rates of coronary interventions. The results of the recent Denmark registry study discussed above suggests that triple therapy provides no additional efficacy benefit while increasing bleeding when compared to VKA plus SAPT [29]. The WOEST trial results suggest that in patients on chronic OAC undergoing PCI, double therapy with warfarin and clopidogrel could be a preferred strategy with respect to mortality and bleeding when compared to TOAT with warfarin, clopidogrel, and aspirin. Thus, the WOEST results contribute further to the concept that OAC plus SAPT might be preferable to TOAT in patients with AF after PCI.

In WOEST, the increase in non-major TIMI bleeding seen with triple therapy was driven largely by gastrointestinal bleeding, skin 
hematomas, and bleeding relating to access sites. Notably, proton pump inhibitors (PPIs) were used in just $25-27 \%$ of patients, and radial access during PCI was performed in only 34-39\%; the authors did not encourage the use of PPIs or radial access in an effort to reproduce a more generalizable study population. The reduction in bleeding seen with double therapy was isolated to TIMI minimal and minor bleeding. However, as the authors mention, non-major bleeding episodes are clinically relevant and often lead to further complications, such as the thrombotic consequences of stopping antithrombotic therapy in response to bleeding.

The WOEST authors chose to exclude aspirin rather than clopidogrel in the double therapy group due to concern that stent thrombosis may be unacceptably increased when dropping clopidogrel. The Denmark registry study grouped VKA plus SAPT in the analysis without differentiating the choice of SAPT, but did note that an analysis of the subset of patients who received VKA plus clopidogrel yielded a bleeding risk similar to that with triple therapy. As such, there is uncertainty regarding the ideal single antiplatelet agent and further investigation is needed before declaring one strategy superior to another. To that end, a trial of patients with OAC undergoing PCI that includes arms of warfarin plus clopidogrel and warfarin plus aspirin would be useful. Prasugrel and ticagrelor also deserve mention in this setting; while the increase in bleeding associated with these agents raises concern for their use in triple therapy, it would be informative to study their efficacy as part of an OAC plus SAPT regimen in this patient population.

Caution is warranted in interpreting the WOEST efficacy results; the study was designed to evaluate safety, not efficacy, and while the composite secondary endpoint met statistical significance, the individual components of MI, stroke, and stent thrombosis did not. The concept of dual therapy actually reducing ischemic and thrombotic events, such as stent thrombosis, is contrary to prior data; given the relatively small study population, such a conclusion should be supported by additional data.

For now, the approaches outlined by the ESC, ACCP, and Circulation authors offer guidance regarding TOAT, and the flow diagram in Fig. 3 above provides an approach to choose antithrombotic therapies. Based on the WOEST results, for the subset of patients at moderate to high risk of stroke, high risk of bleeding, and low risk of stent thrombosis, in whom the Circulation authors, for example, currently recommend one month of TOAT followed by 11 months of VKA plus SAPT, VKA plus SAPT for all 12 months may be a reasonable alternative. However, additional studies are needed to substantiate this approach. For patients at high risk of stent thrombosis, the WOEST trial does not adequately quell concerns that the risk of stent thrombosis will not be unacceptably high if one antiplatelet agent is dropped, and in these patients, a short period of TOAT is probably still reasonable. Regardless of the pharmacological approach, these patients at high risk of both stroke and stent thrombosis should receive bare metal stents whenever feasible.

Lastly, the role of newer anticoagulant agents in TOAT deserves mention, although a full discussion of this topic is beyond the scope of this paper. Dabigatran, rivaroxaban, and apixaban are the three new FDA-approved agents for stroke prevention in non-valvular atrial fibrillation, and these agents were shown to have favorable safety profiles when compared to warfarin, all leading to significant reductions in hemorrhagic stroke [33-35]. These agents, in varying doses, have been studied in ACS patients treated with background dual 
antiplatelet therapy; however, subjects in these trials did not have an indication for chronic full-dose OAC (e.g., AF) [36-39]. As such, further investigation is needed to determine what role these agents may play in TOAT.

\section{CONCLUSION}

Patients on chronic OAC who undergo PCI challenge clinicians to choose the antithrombotic strategy that best balances their unique set of competing risks. Only a few formal recommendations have been published on this issue and all advise using TOAT in some patients for 1-6 months after PCI in an effort to maximize reductions of stroke and stent thrombosis. Based on available data, TOAT is assumed to provide an efficacy benefit that is superior to dual therapy and that sufficiently outweighs any increased bleeding risk of TOAT.

The WOEST trial was a RCT designed to address this issue. The results provide new groundwork for the assumption that triple therapy does yield more bleeding, while challenging prior assumptions of the efficacy benefit of TOAT. As discussed, caution should be taken with this data, awaiting further studies prior to changing practice, and only applying the results to the proper population. In time, these results may impact clinical practice and set the stage for further trials in this area.

\section{ACKNOWLEDGMENTS}

Dr. Mega is the guarantor for this article, and takes responsibility for the article as a whole.

Conflict of interest. Dr. Mega has received grant/research funding from Accumetrics, Bayer Healthcare, BMS, Daiichi Sankyo, Eli Lilly, Johnson \& Johnson, Nanosphere, and Sanofi- aventis and honoraria from Merck, Janssen, and American Genomics. Dr. Carreras has no disclosures.

Open Access. This article is distributed under the terms of the Creative Commons Attribution Noncommercial License which permits any noncommercial use, distribution, and reproduction in any medium, provided the original author(s) and the source are credited.

\section{REFERENCES}

1. Go AS, Mozaffarian D, Roger VL, et al. Heart disease and stroke statistics-2013 update a report from the American Heart Association. Circulation. 2012;127: e6-245.

2. Faxon DP, Eikelboom JW, Berger PB, et al. Antithrombotic therapy in patients with atrial fibrillation undergoing coronary stenting: a North American perspective: executive summary. Circ Cardiovasc Interv. 2011;4:522-34.

3. You JJ, Singer DE, Howard PA, et al. Antithrombotic therapy for atrial fibrillation: antithrombotic therapy and prevention of thrombosis, 9th edn. American College of Chest Physicians evidencebased clinical practice guidelines. Chest. 2012;141(Suppl 2):e531S-75S.

4. Gage BF, Waterman AD, Shannon W, Boechler M, Rich MW, Radford MJ. Validation of clinical classification schemes for predicting stroke: results from the national registry of atrial fibrillation. JAMA. 2001;285:2864-70.

5. Connolly S, Pogue J, Hart R, et al. Clopidogrel plus aspirin versus oral anticoagulation for atrial fibrillation in the atrial fibrillation clopidogrel trial with irbesartan for prevention of vascular events (active $\mathrm{w}$ ): a randomised controlled trial. Lancet. 2006;367:1903-12.

6. Hart RG, Pearce LA, Aguilar MI. Meta-analysis: antithrombotic therapy to prevent stroke in patients who have nonvalvular atrial fibrillation. Ann Intern Med. 2007;146:857-67.

7. Lloyd-Jones DM, Wang TJ, Leip EP, et al. Lifetime risk for development of atrial fibrillation: the framingham heart study. Circulation. 2004;110: 1042-6. 
8. [No Authors Listed.] Collaborative overview of randomised trials of antiplatelet therapy-I: prevention of death, myocardial infarction, and stroke by prolonged antiplatelet therapy in various categories of patients. Antiplatelet trialists' collaboration. BMJ. 1994;308:81-106.

9. Yusuf S, Zhao F, Mehta SR, Chrolavicius S, Tognoni G, Fox KK. Effects of clopidogrel in addition to aspirin in patients with acute coronary syndromes without st-segment elevation. NEJM. 2001;345:494-502.

10. Leon MB, Baim DS, Popma JJ, et al. A clinical trial comparing three antithrombotic-drug regimens after coronary-artery stenting. Stent anticoagulation restenosis study investigators. NEJM. 1998;339:1665-71.

11. Wallentin L, Becker RC, Budaj A, et al. Ticagrelor versus clopidogrel in patients with acute coronary syndromes. NEJM. 2009;361:1045-57.

12. Wiviott SD, Braunwald E, McCabe $\mathrm{CH}$, et al. Prasugrel versus clopidogrel in patients with acute coronary syndromes. NEJM. 2007;357:2001-15.

13. Park SJ, Park DW, Kim YH, et al. Duration of dual antiplatelet therapy after implantation of drugeluting stents. NEJM. 2010;362:1374-82.

14. Valgimigli M, Campo G, Percoco G, et al. Randomized comparison of 6- versus 24-month clopidogrel therapy after balancing anti-intimal hyperplasia stent potency in all-comer patients undergoing percutaneous coronary intervention design and rationale for the prolonging dualantiplatelet treatment after grading stent-induced intimal hyperplasia study (prodigy). Am Heart J. 2010;160:804-11.

15. Byrne RA, Schulz S, Mehilli J, et al. Rationale and design of a randomized, double-blind, placebocontrolled trial of 6 versus 12 months clopidogrel therapy after implantation of a drug-eluting stent: the intracoronary stenting and antithrombotic regimen: safety and efficacy of 6 months dual antiplatelet therapy after drug-eluting stenting (ISAR-SAFE) study. Am Heart J. 2009;157:620-4.

16. Lemesle G, Paparoni F, Delhaye C, Bonello L, Lablanche JM. Duration of dual antiplatelet therapy after percutaneous coronary intervention with drug-eluting stent implantation: a review of the current guidelines and literature. Hosp Pract (Minneap). 2011;39:32-40.

17. Rothberg MB, Celestin C, Fiore LD, Lawler E, Cook JR. Warfarin plus aspirin after myocardial infarction or the acute coronary syndrome: meta-analysis with estimates of risk and benefit. Ann Intern Med. 2005;143:241-50.
18. Andreotti F, Testa L, Biondi-Zoccai GG, Crea F. Aspirin plus warfarin compared to aspirin alone after acute coronary syndromes: an updated and comprehensive meta-analysis of 25,307 patients. Eur Heart J. 2006;27:519-26.

19. Hurlen $M$, Abdelnoor $M$, Smith $P$, Erikssen J, Arnesen H. Warfarin, aspirin, or both after myocardial infarction. NEJM. 2002;347:969-74.

20. van Es RF, Jonker JJ, Verheugt FW, Deckers JW, Grobbee DE. Aspirin and coumadin after acute coronary syndromes (the aspect-2 study): a randomised controlled trial. Lancet. 2002;360:109-13.

21. Ruiz-Nodar JM, Marin F, Hurtado JA, et al. Anticoagulant and antiplatelet therapy use in 426 patients with atrial fibrillation undergoing percutaneous coronary intervention and stent implantation implications for bleeding risk and prognosis. J Am Coll Cardiol. 2008;51:818-25.

22. Khurram Z, Chou E, Minutello $\mathrm{R}$, et al. Combination therapy with aspirin, clopidogrel and warfarin following coronary stenting is associated with a significant risk of bleeding. J Invasive Cardiol. 2006;18:162-4.

23. DeEugenio D, Kolman L, DeCaro M, et al. Risk of major bleeding with concomitant dual antiplatelet therapy after percutaneous coronary intervention in patients receiving long-term warfarin therapy. Pharmacotherapy. 2007;27:691-6.

24. Karjalainen PP, Porela P, Ylitalo A, et al. Safety and efficacy of combined antiplatelet-warfarin therapy after coronary stenting. Eur Heart J. 2007;28:726-32.

25. Sarafoff N, Ndrepepa G, Mehilli J, et al. Aspirin and clopidogrel with or without phenprocoumon after drug eluting coronary stent placement in patients on chronic oral anticoagulation. J Intern Med. 2008;264:472-80.

26. Rossini R, Musumeci G, Lettieri C, et al. Long-term outcomes in patients undergoing coronary stenting on dual oral antiplatelet treatment requiring oral anticoagulant therapy. Am J Cardiol. 2008; 102:1618-23.

27. Holmes DR Jr, Kereiakes DJ, Kleiman NS, Moliterno DJ, Patti G, Grines CL. Combining antiplatelet and anticoagulant therapies. J Am Coll Cardiol. 2009;54:95-109.

28. Lip GY, Huber K, Andreotti F, et al. Antithrombotic management of atrial fibrillation patients presenting with acute coronary syndrome and/or undergoing coronary stenting: executive summary-a consensus document of the european 
society of cardiology working group on thrombosis, endorsed by the european heart rhythm association (ehra) and the european association of percutaneous cardiovascular interventions (eapci). Eur Heart J. 2010;31:1311-8.

29. Lamberts M, Olesen JB, Ruwald MH, et al. Bleeding after initiation of multiple antithrombotic drugs, including triple therapy, in atrial fibrillation patients following myocardial infarction and coronary intervention: a nationwide cohort study. Circulation. 2012;126:1185-93.

30. Dewilde W, Berg JT. Design and rationale of the WOEST trial: What is the optimal antiplatelet and anticoagulant therapy in patients with oral anticoagulation and coronary stenting (WOEST). Am Heart J. 2009;158:713-8.

31. Dewilde $\mathrm{W}$, Oirbans $\mathrm{T}$, Verheugt $\mathrm{F}$, et al. The WOEST trial-clinical trial results. Paper presented at: European Society of Cardiology Congress; Aug 28, 2012; Munich, Germany.

32. Dewilde WJ, Oirbans T, Verheugt FW, et al. Use of clopidogrel with or without aspirin in patients taking oral anticoagulant therapy and undergoing percutaneous coronary intervention: an open-label, randomised, controlled trial. Lancet. 2013;381: 1107-15.

33. Patel MR, Mahaffey KW, Garg J, et al. Rivaroxaban versus warfarin in nonvalvular atrial fibrillation. NEJM. 2011;365:883-91.
34. Connolly SJ, Ezekowitz MD, Yusuf S, et al. Dabigatran versus warfarin in patients with atrial fibrillation. NEJM. 2009;361:1139-51.

35. Granger CB, Alexander JH, McMurray JJ, et al. Apixaban versus warfarin in patients with atrial fibrillation. NEJM. 2011;365:981-92.

36. Alexander JH, Becker RC, Bhatt DL, et al. Apixaban, an oral, direct, selective factor Xa inhibitor, in combination with antiplatelet therapy after acute coronary syndrome: Results of the apixaban for prevention of acute ischemic and safety events (APPRAISE) trial. Circulation. 2009;119:2877-85.

37. Alexander JH, Lopes RD, James S, et al. Apixaban with antiplatelet therapy after acute coronary syndrome. NEJM. 2011;365:699-708.

38. Mega JL, Braunwald E, Mohanavelu S, et al. Rivaroxaban versus placebo in patients with acute coronary syndromes (ATLAS ACS-TIMI 46): a randomised, double-blind, phase ii trial. Lancet. 2009;374:29-38.

39. Mega JL, Braunwald E, Wiviott SD, et al. Rivaroxaban in patients with a recent acute coronary syndrome. NEJM. 2012;366:9-19. 